Revista Arbitrada Interdisciplinaria de Ciencias de la Salud. SALUD Y VIDA

Volumen 4. Número 8. Año 4. Julio - Diciembre 2020

Hecho el depósito de Ley: FA2016000010

ISSN: 2610-8038

FUNDACIÓN KOINONIA (F.K).

Santa Ana de Coro, Venezuela.

Franklin Antonio Vite-Solorzano; Leobaldo Barrera-Paz; Jefferson Alexander Sornoza-Párraga Angy Vanessa Salazar-Morrillo

http://dx.doi.org/10.35381/s.v.v4i8.1011

\title{
Autocuidado, condicionantes y evolución de los pacientes con diabetes mellitus tipo 2
}

\section{Self-care, conditioning factors and evolution of patients with type 2 diabetes mellitus}

Franklin Antonio Vite-Solorzano antuanvit@hotmail.com

Universidad Técnica de Manabí, Portoviejo

Ecuador

https://orcid.org/0000-0003-0236-0002

Leobaldo Barrera-Paz

depgfmluz@gmail.com

Universidad del Zulia, Maracaibo

https://orcid.org/0000-0003-4480-0416

Jefferson Alexander Sornoza-Párraga

alexanderptx@gmail.com

Universidad Técnica de Manabí, Portoviejo

Ecuador

https://orcid.org/0000-0001-6709-5612

Angy Vanessa Salazar-Morrillo

vanessa angy.97@outlook.com

Universidad Técnica de Manabí, Portoviejo

Ecuador

https://orcid.org/0000-0002-2088-606X

Recepción: 15 de junio 2020

Revisado: 28 de julio 2020

Aprobación: 25 de agosto 2020

Publicación: 03 de septiembre 2020 


\title{
RESUMEN
}

Objetivo: estudiar la relación entre los factores básicos condicionantes y la evolución de la enfermedad en el autocuidado de los pacientes con diabetes mellitus tipo 2. Metodología: De tipo cuantitativo - descriptivo y transversal, la población estuvo constituida por 90 pacientes con diabetes mellitus tipo 2 que viven en el contexto rural de la provincia de Manabí - Ecuador y cumplieron con los siguientes criterios de inclusión, pacientes mayores de 18 años de edad de los subcentros de salud de Manabí, sin discriminación por género, edad, etnia, tiempo laboral o nivel de formación. Además, la aceptación y firma del consentimiento informado. Conclusión: Existe un total acuerdo entre los pacientes investigados, en considerar que poseen una buena capacidad de agencia de autocuidado, a pesar que existe una relación de dependencia significativa con los factores condicionantes de la edad, sexo, estado civil y evolución de la enfermedad.

Descriptores: Diabetes mellitus; intolerancia a la glucosa; metabolismo. (Fuente: DeCS2020).

\begin{abstract}
Objective: to study the relationship between the basic conditioning factors and evolution of the disease in the self-care of patients with type 2 diabetes mellitus. Methodology: Of a descriptive and cross-cutting quantitative type, the population consisted of 90 patients with type 2 diabetes mellitus living in the rural context of manabí province - Ecuador and met the following criteria of inclusion, patients over 18 years of age, of the health subcenters of Manabí, without discrimination by gender, age, ethnicity, working time or level of training. In addition, the acceptance and signature of informed consent. Conclusion: There is a total agreement among the investigated patients, in considering that they have a good capacity as a self-care agency, even though there is a significant relationship of dependence with the conditioning factors of age, sex, marital status and evolution of the disease.
\end{abstract}

Descriptors: Diabetes mellitus; glucose intolerance; metabolism. (Source: DeCS2020). 
Revista Arbitrada Interdisciplinaria de Ciencias de la Salud. SALUD Y VIDA

Volumen 4. Número 8. Año 4. Julio - Diciembre 2020

Hecho el depósito de Ley: FA2016000010

ISSN: 2610-8038

FUNDACIÓN KOINONIA (F.K).

Santa Ana de Coro, Venezuela.

Franklin Antonio Vite-Solorzano; Leobaldo Barrera-Paz; Jefferson Alexander Sornoza-Párraga Angy Vanessa Salazar-Morrillo

\section{INTRODUCCIÓN}

El autocuidado son las acciones intencionadas de las personas para controlar sus factores internos o externos, que pueden comprometer su vida y desarrollo posterior. La capacidad de agencia de autocuidado, que deben desarrollar los individuos con diabetes es fundamental, por cuanto permite prevenir las complicaciones desencadenadas por un manejo inadecuado de la enfermedad ${ }^{12}$.

Al respecto, las investigaciones sobre diabetes demuestran que más de la mitad de los pacientes manifiestan estilos de vida no saludables, como incumplimiento de la dieta y de la práctica de ejercicios físicos, falta de control metabólico, el abuso de la ingestión de sustancias psicótropas, así como insuficiente conocimiento de su enfermedad. Por tanto, se hace necesario el desarrollo de investigaciones que indaguen en el autocuidado que presentan estos pacientes en función del posterior desarrollo de programas de intervención que promuevan el autocuidado ${ }^{345}$.

Todo ello, ante la diversidad conceptual del autocuidado dentro de contextos culturales y sociales específicos. En los países del continente Americano, el autocuidado se refiere a las acciones que las personas adoptan en beneficio de su propia salud, sin supervisión médica formal; éste es definido como prácticas realizadas por las personas y familias mediante las cuales son promovidos comportamientos positivos de salud, en la prevención de enfermedades y en el tratamiento de los síntomas 678 .

Por otra parte, se destaca el hecho en la cual la Organización Mundial de la Salud recomienda la educación para el autocuidado con la finalidad de prevenir y tratar las enfermedades crónicas 9 10. La educación para el autocuidado de personas con problemas crónicos de salud debe promover el soporte para el desarrollo de las habilidades de autocuidado, a fin de responsabilizarlas por su salud y ayudarlas a aprender a convivir mejor con la enfermedad, modificar o mantener los hábitos saludables, estimular la autoconfianza para sentirse mejor cualquiera que sea la gravedad de la enfermedad ${ }^{11}$. 
Franklin Antonio Vite-Solorzano; Leobaldo Barrera-Paz; Jefferson Alexander Sornoza-Párraga Angy Vanessa Salazar-Morrillo

En el caso de la diabetes mellitus tipo 2, las complicaciones en los distintos órganos puede incluso llevar a discapacidad o a la muerte del paciente. En este caso, el personal de salud debe estar preparado y presto para orientar al paciente diabético en su responsabilidad de autocuidado para evitar las complicaciones. Ello, sin descuidar sus responsabilidades clínicas en el examen exhaustivo del estado físico de los mismos, lo que permitiría detectar precozmente si se presenta alguna de las complicaciones mencionadas y así limitar tanto los daños como las consecuencias negativas que podría significar el avance del deterioro de las condiciones en la supervivencia y la calidad de vida de los pacientes 12131415 .

Al respecto, es de indicar que el autocuidado de los pacientes con diabetes mellitus tipo 2, permite su desarrollo de la capacidad de agencia de autocuidado, para asi prevenir las complicaciones que se pueden presentar por un manejo inadecuado de la enfermedad. Para ello, se plantean 161718 tres elementos que deben considerar durante el afianzamiento de las habilidades que requieren para cuidar su salud, entre estos elementos se destacan:

Capacidades fundamentales y disposición del autocuidado: consideradas como las habilidades básicas del individuo y refieren a la percepción, memoria y orientación que deben tomar en cuenta durante su autocuidado.

Componentes de poder: habilidades que impulsan al paciente, la acción de autocuidado a través de la motivación, adquisición de conocimientos, habilidad para ordenar acciones de autocuidado y capacidad de integración del autocuidado en su vida familiar y comunitaria.

Capacidad de operacionalizar el autocuidado: habilidades que permiten al paciente, investigar sobre condiciones de sí mismo y del medio ambiente, que son significativos para su autocuidado, así como la toma de decisiones y la construcción de las acciones para lograr el manejo de su enfermedad. 
Revista Arbitrada Interdisciplinaria de Ciencias de la Salud. SALUD Y VIDA

Volumen 4. Número 8. Año 4. Julio - Diciembre 2020

Hecho el depósito de Ley: FA2016000010

ISSN: 2610-8038

FUNDACIÓN KOINONIA (F.K)

Santa Ana de Coro, Venezuela.

Franklin Antonio Vite-Solorzano; Leobaldo Barrera-Paz; Jefferson Alexander Sornoza-Párraga

Angy Vanessa Salazar-Morrillo

\section{Factores básicos condicionantes de la enfermedad en pacientes con Diabetes mellitus 2}

A pesar de ser la diabetes una condición metabólica muy conocida, aún se desconocen las causas exactas de la misma, lo que sí está comprobado que existen algunos factores de riesgo para padecerla 192021 , entre las que se destacan:

La obesidad, apoyada en una característica común al contar con un alto porcentaje de más del $60 \%$ de los diabéticos poseen sobrepeso, donde la sensibilidad de las células pancreáticas de las células $\beta$ a los niveles elevados de glucosa en sangre disminuye, y la cantidad de receptores de insulina disminuye en las células del tejido diana, principalmente en las células musculares, lo que trae como consecuencia una reducción en la "entrega" de glucosa a las células y un aumento en su concentración en sangre ${ }^{22}$. El embarazo, es otro factor condicionante, debido que se da un aumento en la cantidad de ácidos grasos y hormonas en la sangre, generándose una disminución de la sensibilidad a la insulina, al mismo tiempo que aumenta su degradación bajo la influencia de la hormona de la placenta ${ }^{23}$.

Medicamentos y agentes químicos, se han considerados dos grupos, el primer grupo de medicamentos incluye medicamentos utilizados para enfermedades reumáticas, respiratorias crónicas, renales y de la piel y diversas afecciones alérgicas. El segundo grupo de medicamentos son preparaciones que actúan sobre la presión arterial elevada y la masa muscular aumentada. Entre otros medicamentos los anticonceptivos orales, para evitarlo se debe controlar su uso de manera oportuna para corregir un trastorno del nivel de azúcar en la sangre.

Otros factores, es de señalar los factores están condicionados al desarrollo de la enfermedad, la cual, es causada por una disfunción de las células ß y/o unos factores de riesgo como la edad, sobrepeso, historia familiar de diabetes, inactividad física, historia de diabetes gestacional, hipertensión arterial, colesterol HDL $\leq 35 \mathrm{mg} / \mathrm{dl}$ o triglicéridos $\geq$ $250 \mathrm{mg} / \mathrm{dl}$, entre otros. 
Franklin Antonio Vite-Solorzano; Leobaldo Barrera-Paz; Jefferson Alexander Sornoza-Párraga Angy Vanessa Salazar-Morrillo

\section{Tiempo de evolución de la enfermedad en pacientes con Diabetes mellitus 2}

El tratamiento de la diabetes en los adultos se centra en mantener controlado durante el máximo tiempo posible el índice de glucemia en sangre para evitar daños colaterales, ya que estos incluso pueden llegar a originar la muerte del afectado. Algunos de las complicaciones que genera la diabetes son 24: daños en los nervios, enfermedades oculares, problemas de circulación, problemas renales, complicaciones en los pies ${ }^{25} \mathrm{y}$ problemas dentales.

Estos problemas se agravan aún más cuando el paciente diabético es una persona mayor, y con cierto grado de deterioro funcional, ya que merman su autonomía y favorecen la dependencia. Es por ello que los tratamientos para la diabetes en la tercera edad, no sólo se centran en mantener controlado el índice glucémico, sino también en prevenir estos problemas que impactan directamente en su calidad de vida ${ }^{26}$.

En este sentido, los pacientes pueden asumir un rol activo que se caracterice por seguir las pautas educativas sugeridas por su médico tratante y por las enfermeras o hacer caso omiso lo que contribuiría al deterioro de su estado de salud, en atención a ello se propone en este artículo estudiar la relación entre los factores básicos condicionantes y evolución de la enfermedad en el autocuidado de los pacientes con diabetes mellitus tipo 2.

\section{METODOLOGIA}

\section{Tipo y diseño de investigación}

El tipo de estudio fue observacional descriptivo y transversal.

\section{Muestra poblacional}

La muestra se integró por 90 pacientes con diabetes mellitus tipo 2 que viven en el contexto rural de la provincia de Manabí - Ecuador. 
Franklin Antonio Vite-Solorzano; Leobaldo Barrera-Paz; Jefferson Alexander Sornoza-Párraga Angy Vanessa Salazar-Morrillo

\section{Criterios de inclusión}

Pacientes mayores de 18 años de edad de los subcentros de salud de Manabí, sin discriminación por género, edad, etnia, tiempo laboral o nivel de formación. Además, la aceptación y firma del consentimiento informado.

\section{Instrumentos de recolección de información}

Las variables del estudio se midieron a través de dos instrumentos: 1) Cuestionario de Factores básicos condicionantes de tipo sociodemograficos, con una escala dicotómica, el cual constó de 8 características referidas a la edad, sexo, estado civil, número de hijos, vivienda, participación social, religión y situación académica.

El segundo instrumento identifica la opinión de los pacientes y el desarrollo de capacidades de autocuidado, se incluyen 24 preguntas con opciones de respuesta tipo Likert: (totalmente de acuerdo (1), de acuerdo (2), no sé (3) en desacuerdo (4), totalmente en desacuerdo (5).

\section{Análisis de datos}

Los datos fueron analizados por medio del paquete estadístico para las ciencias sociales SPSS versión 23. De cada variable se obtuvieron estadísticas descriptivas (frecuencia, porcentajes, media y desviación estándar) y estadísticas inferenciales. Se aplicó el coeficiente de correlación de Chi cuadrado para evaluar la relación de dependencias entre las variables estudiadas. Los resultados se presentaron en tablas con sus respectivos análisis. 
Revista Arbitrada Interdisciplinaria de Ciencias de la Salud. SALUD Y VIDA

Volumen 4. Número 8. Año 4. Julio - Diciembre 2020

Hecho el depósito de Ley: FA2016000010

ISSN: $2610-8038$

FUNDACIÓN KOINONIA (F.K).

Santa Ana de Coro, Venezuela.

Franklin Antonio Vite-Solorzano; Leobaldo Barrera-Paz; Jefferson Alexander Sornoza-Párraga Angy Vanessa Salazar-Morrillo

\section{RESULTADOS}

En esta sección del artículo, se diagnostican los resultados obtenidos por medio de la aplicación de los instrumentos; escala de valoración de la capacidad de agencia de autocuidado ASA, Factores básicos condicionantes de tipo sociodemograficos y tiempo de evolución de la enfermedad en pacientes con Diabetes mellitus 2, cuyos datos fueron procesados estadísticamente y se presentan en tablas con sus respectivas interpretaciones.

\section{Tabla 1.}

Valor de la media y desviación estándar (DE) por ítem de la escala de valoración de la capacidad de agencia de autocuidado ASA en pacientes con Diabetes mellitus 2.

\begin{tabular}{|l|c|}
\hline PREGUNTAS & Media \pm DE \\
\hline 1. ¿Hago ajustes en mis hábitos de vida para Mantenerme saludable? & $4,8 \pm 0,35$ \\
\hline 2. Verifico si mis acciones y actitudes me protegen y mantienen mi saluo & $4,6 \pm 0,46$ \\
\hline $\begin{array}{l}\text { 3. ¿Hago cambios o ajustes de mi rutina si tengo problemas para } \\
\text { realizar sus haceres cotidiano }\end{array}$ & $4,6 \pm 0,47$ \\
\hline 4. ¿Hago lo necesario para mantener limpio mi alrededor? & $4,8 \pm 0,37$ \\
\hline $\begin{array}{l}\text { 5. Si es necesario, ¿doy prioridad a las acciones que mantienen y } \\
\text { protegen mi salud? }\end{array}$ & $4,8 \pm 0,35$ \\
\hline 6. ¿Tengo la energía para cuidarme cómo debo? & $4,7 \pm 0,43$ \\
\hline 7. ¿Busco mejores formas de cuidar mi salud? & $4,7 \pm 0,45$ \\
\hline $\begin{array}{l}\text { 8. Con tal de mantenerme limpio, ¿realizo ajustes en cuanto a mi } \\
\text { higiene personal? }\end{array}$ & $4,8 \pm 0,37$ \\
\hline $\begin{array}{l}\text { 9. ¿Me alimento de acuerdo a mi situación de salud y mantengo un pesc } \\
\text { adecuado? }\end{array}$ & $4,7 \pm 0,41$ \\
\hline $\begin{array}{l}\text { 10. ¿Tengo la capacidad de manejar las situaciones para no perder el } \\
\text { control y mantenerme en equilibrio? }\end{array}$ & $4,7 \pm 0,44$ \\
\hline 11. ¿Hago ejercicio y descanso durante el día? & $4,5 \pm 0,49$ \\
\hline 12. ¿Tengo amigos a quienes puedo recurrir cuando necesito ayuda? & $4,6 \pm 0,49$ \\
\hline 13. ¿Puedo dormir lo suficiente para sentirme descansado? & $4,7 \pm 0,45$ \\
\hline $\begin{array}{l}\text { 14. Cuando obtengo información sobre mi salud, ¿pido explicación } \\
\text { sobre lo que no entiendo? }\end{array}$ & $4,8 \pm 0,32$ \\
\hline 15. ¿Reviso mi cuerpo para saber si hay algún cambio? & $4,7 \pm 0,43$ \\
\hline $\begin{array}{l}\text { 16. He sido capaz de cambiar hábitos no beneficiosos con tal de mejora } \\
\text { salud. }\end{array}$ & $4,7 \pm 0,45$ \\
\hline 17. Si tengo que tomar un nuevo medicamento ¿pido información & $4,7 \pm 0,43$ \\
\hline
\end{tabular}


Revista Arbitrada Interdisciplinaria de Ciencias de la Salud. SALUD Y VIDA

Volumen 4. Número 8. Año 4. Julio - Diciembre 2020

Hecho el depósito de Ley: FA2016000010

ISSN: 2610-8038

FUNDACIÓN KOINONIA (F.K).

Santa Ana de Coro, Venezuela.

Franklin Antonio Vite-Solorzano; Leobaldo Barrera-Paz; Jefferson Alexander Sornoza-Párraga Angy Vanessa Salazar-Morrillo

\begin{tabular}{|l|c|}
\hline \multicolumn{2}{|l|}{ sobre sus efectos? } \\
\hline 18. ¿Tomo medidas para asegurar que mi familia y yo estemos seguros & $4,6 \pm 0,46$ \\
\hline 19.- ¿Evalúo qué tan efectivo es lo que hago para mantenerme con salu & $4,8 \pm 0,39$ \\
\hline 20. En mis actividades diarias, ¿me tomo un tiempo para cuidarme? & $4,6 \pm 0,46$ \\
\hline 21. ¿Busco información sobre mi salud si esta se ve afectada? & $4,8 \pm 0,39$ \\
\hline $\begin{array}{l}\text { 22. ¿Busco ayuda si me encuentro con problemas de salud o de otro } \\
\text { tipo? }\end{array}$ & $4,8 \pm 0,32$ \\
\hline 23. ¿Dedico tiempo para mi mismo? & $4,7 \pm 0,44$ \\
\hline 24. Aun con problemas de salud soy capaz de cuidarme & $4,8 \pm 0,38$ \\
\hline
\end{tabular}

Fuente: Elaboración propia (2020).

En la tabla 1, se indica que el total de las opiniones por parte de los investigados, en la que se observa que el $100 \%$ del total se evaluaron con una media por encima de 4 implicando que están totalmente de acuerdo, donde los ítems se indicaron de mayor a menor aceptación de la siguiente orden con una media de 4,8:

¿Hago ajustes en mis hábitos de vida para mantenerme saludable?, ¿Hago lo necesario para mantener limpio mi alrededor?, ¿Si es necesario, doy prioridad a las acciones que mantienen y protegen mi salud?, ¿Con tal de mantenerme limpio, ¿realizo ajustes en cuanto a mi higiene personal? Cuando obtengo información sobre mi salud, ¿pido explicación sobre lo que no entiendo?, ¿Evalúo qué tan efectivo es lo que hago para mantenerme con salud?, ¿Busco información sobre mi salud si esta se ve afectada?, ¿Aun con problemas de salud soy capaz de cuidarme?

Con 4.7: ¿Tengo la energía para cuidarme cómo debo?, ¿Busco mejores formas de cuidar mi salud?, ¿Me alimento de acuerdo a mi situación de salud y mantengo un peso adecuado?, ¿Tengo la capacidad de manejar las situaciones para no perder el control y mantenerme en equilibrio?, ¿Puedo dormir lo suficiente para sentirme descansado?, ¿Reviso mi cuerpo para saber si hay algún cambio?, He sido capaz de cambiar hábitos no beneficiosos con tal de mejorar mi salud, Si tengo que tomar un nuevo medicamento ¿pido información sobre sus efectos?

¿Dedico tiempo para mí mismo?; con 4,6: Verifico si mis acciones y actitudes me 
Revista Arbitrada Interdisciplinaria de Ciencias de la Salud. SALUD Y VIDA

Volumen 4. Número 8. Año 4. Julio - Diciembre 2020

Hecho el depósito de Ley: FA2016000010

ISSN: 2610-8038

FUNDACIÓN KOINONIA (F.K).

Santa Ana de Coro, Venezuela.

Franklin Antonio Vite-Solorzano; Leobaldo Barrera-Paz; Jefferson Alexander Sornoza-Párraga Angy Vanessa Salazar-Morrillo

protegen y mantienen mi salud, ¿Hago cambios o ajustes de mi rutina si tengo problemas para realizar sus haceres cotidiano, ¿Tengo amigos a quienes puedo recurrir cuando necesito ayuda?, ¿Tomo medidas para asegurar que mi familia y yo estemos seguros?, en mis actividades diarias, ¿me tomo un tiempo para cuidarme? Con la menor media de aceptación se presentó el ítem ¿Hago ejercicio y descanso durante el día?

Tabla 2.

Factores básicos condicionantes de tipo sociodemograficos en pacientes con Diabetes mellitus 2.

\begin{tabular}{|c|c|c|c|c|}
\hline Característica & Indicador & $\mathrm{Fr}$ & $\%$ & Media \pm DE \\
\hline \multirow[t]{6}{*}{ Edad } & $30-40$ años & 6 & 7 & \multirow{6}{*}{$3,3667 \pm, 13996$} \\
\hline & $41-50$ años & 21 & 23 & \\
\hline & $51-60$ años & 23 & 26 & \\
\hline & $61-70$ años & 17 & 19 & \\
\hline & $71-80$ años & 20 & 22 & \\
\hline & 81-90 años & 3 & 3 & \\
\hline \multirow[t]{2}{*}{ Sexo } & Femenino & 49 & 54 & \multirow{2}{*}{$1,4556 \pm, 05279$} \\
\hline & Masculino & 41 & 46 & \\
\hline \multirow[t]{6}{*}{ Estado civil } & Soltero/a & 6 & 7 & \multirow{6}{*}{$3,4333 \pm, 12143$} \\
\hline & Comprometido/a & 0 & 0 & \\
\hline & Casado/a & 55 & 61 & \\
\hline & Unión de hecho & 14 & 15 & \\
\hline & Separado/a & 7 & 8 & \\
\hline & Viudo/a & 8 & 9 & \\
\hline \multirow[t]{3}{*}{ Número de hijos } & 1 a 5 hijos & 66 & 73 & \multirow{3}{*}{$1,3556 \pm, 06762$} \\
\hline & 6 a 10 & 16 & 18 & \\
\hline & $>10$ hijos & 8 & 9 & \\
\hline \multirow[t]{3}{*}{ Vivienda } & Casa de algún familiar & 33 & 37 & \multirow{3}{*}{$2,0222 \pm, 09211$} \\
\hline & Casa alquiler & 21 & 23 & \\
\hline & Casa propia & 36 & 40 & \\
\hline \multirow[t]{3}{*}{ Participación social } & $\begin{array}{lll}\begin{array}{l}\text { Equipo } \\
\text { deportiva }\end{array} & 0 & \text { agrupación } \\
\end{array}$ & 21 & 23 & \multirow[t]{3}{*}{$2,3444 \pm, 08821$} \\
\hline & Grupo religioso & 17 & 19 & \\
\hline & Club diabético & 52 & 58 & \\
\hline \multirow[t]{3}{*}{ Religión } & Católica & 58 & 65 & \multirow{3}{*}{$2,4111 \pm, 08924$} \\
\hline & Evangélico & 21 & 23 & \\
\hline & Otro & 11 & 12 & \\
\hline Situación & Primario completa & 54 & 60 & $1,7667 \pm, 11290$ \\
\hline
\end{tabular}


Revista Arbitrada Interdisciplinaria de Ciencias de la Salud. SALUD Y VIDA

Volumen 4. Número 8. Año 4. Julio - Diciembre 2020

Hecho el depósito de Ley: FA2016000010

ISSN: $2610-8038$

FUNDACIÓN KOINONIA (F.K).

Santa Ana de Coro, Venezuela.

Franklin Antonio Vite-Solorzano; Leobaldo Barrera-Paz; Jefferson Alexander Sornoza-Párraga Angy Vanessa Salazar-Morrillo

\begin{tabular}{|l|l|l|l|l|}
\hline \multirow{3}{*}{ académica } & Secundario completa & 13 & 14,5 & \multirow{2}{*}{} \\
\cline { 2 - 4 } & Superior & 13 & 14,5 & \\
\cline { 2 - 4 } & Ninguno & 10 & 11 & \\
\hline
\end{tabular}

Fuente: Elaboración propia (2020).

En la tabla 2, se indican los factores básicos condicionantes de tipo sociodemográfico, donde se observa el condicionante edad de los pacientes investigados con una media de 3,3667 ubicando su predominio con un $26 \%$ del total en edades comprendidas entre 51 y 60 años, el 54\% son de sexo femenino. El estado civil con una media de 3,4333 ubica con el $61 \%$ del total en la condición casado/a, y el 63\% tienen entre 1 a 5 hijos. Por otra parte, se indica que el $770 \%$ poseen casa propia o viven con algún familiar. Tambien, expreso el $52 \%$ de los pacientes pertenecer al club de los diabéticos y un $65 \%$ practicar la religión católica. Se indica que el 60 \% alcanzo solo culminar la educación primaria.

\section{Tabla 3.}

Tiempo de evolución de la enfermedad en pacientes con Diabetes mellitus 2.

\begin{tabular}{|c|l|l|l|}
\hline Tiempo/estadística & FR & $\%$ & \\
\hline De 1 a 5 años & $\mathbf{2 1}$ & $\mathbf{2 3}$ & \\
\hline 5 a 10 años & $\mathbf{3 6}$ & $\mathbf{4 0}$ & \\
& & & \\
\hline 10 a 20 años & $\mathbf{2 7}$ & $\mathbf{3 0}$ & \\
\hline Más de 20 años & $\mathbf{6}$ & $\mathbf{7}$ & \\
\hline Media \pm DE & $\mathbf{2 , 2} \pm 0,87$ & & \\
\hline
\end{tabular}

Fuente: Elaboración propia (2020).

En la tabla 3, se indica el tiempo de evolución de la enfermedad en pacientes con Diabetes mellitus 2, en la que se observa que la media de 2, 2 verifica que la mayor concentración de los pacientes está dada entre 5 a 10 años de evolución de la enfermedad representado con el $40 \%$ del total los investigados. Seguido del $30 \%$ que poseen la enfermedad entre el lapso de 
Franklin Antonio Vite-Solorzano; Leobaldo Barrera-Paz; Jefferson Alexander Sornoza-Párraga Angy Vanessa Salazar-Morrillo

10 a 20 años. Los menores porcentajes se indicador para pacientes que tienen entre 1 a 5 años con el $23 \%$ y solo el $7 \%$ señalo tener más de 20 años de tiempo de evolución de la enfermedad.

\section{Tabla 4.}

Relación Chi cuadrado entre los Factores Básicos Condicionantes de Tipo Sociodemográficos y la variable capacidad de agencia de autocuidado ASA en pacientes con Diabetes mellitus 2.

\begin{tabular}{|l|l|l|}
\hline $\begin{array}{l}\text { Factores/ } \\
\text { Autocuidado ASA chi }\end{array}$ & Valor Chi & Valor P \\
\hline Edad & 0,000 & 0,05 \\
\hline Sexo & 0,000 & $\mathbf{0 , 0 5}$ \\
\hline Estado civil & $\mathbf{0 , 0 0 0}$ & $\mathbf{0 , 0 5}$ \\
\hline Número de hijos & $\mathbf{1 , 0 0 0}$ & $\mathbf{0 , 0 5}$ \\
\hline Vivienda & $\mathbf{0 , 9 9 9}$ & $\mathbf{0 , 0 5}$ \\
\hline Participación social & $\mathbf{0 , 0 0 0}$ & $\mathbf{0 , 0 5}$ \\
\hline Religión & $\mathbf{0 , 0 9 0}$ & $\mathbf{0 , 0 5}$ \\
\hline Situación académica & $\mathbf{1 , 0 0 0}$ & $\mathbf{0 , 0 5}$ \\
\hline
\end{tabular}

Fuente: Elaboración propia (2020).

En la tabla 4, se presenta el análisis chi cuadrado entre la variable capacidad de agencia de autocuidado ASA y los Factores Básicos Condicionantes de Tipo Sociodemográficos en pacientes con Diabetes mellitus 2, en la que se indica que el valor chi calculado con un valor de 0.000 de los factores condicionantes de la edad, sexo, estado civil y participación social es menor que el valor chi de la tabla con 0.05 , implicando que existe dependencia entre las variables, lo que evidencia que la variable capacidad de agencia del autocuidado está siendo afectada por dichos factores. Mientras que los factores números de hijos, vivienda, religión y situación académica no presentaron relación de dependencia con el autocuidado. 
Revista Arbitrada Interdisciplinaria de Ciencias de la Salud. SALUD Y VIDA

Volumen 4. Número 8. Año 4. Julio - Diciembre 2020

Hecho el depósito de Ley: FA2016000010

ISSN: $2610-8038$

FUNDACIÓN KOINONIA (F.K)

Santa Ana de Coro, Venezuela.

Franklin Antonio Vite-Solorzano; Leobaldo Barrera-Paz; Jefferson Alexander Sornoza-Párraga

Angy Vanessa Salazar-Morrillo

\section{Tabla 5.}

Relación Chi cuadrado entre el Tiempo de evolución de la enfermedad y la variable capacidad de agencia de autocuidado ASA en pacientes con Diabetes mellitus 2.

\begin{tabular}{lc} 
Análisis / Posición & Valor \\
\hline \hline VALOR P & 0.05 \\
\hline VALOR CHI & 0.000 \\
\hline
\end{tabular}

Fuente: Elaboración propia (2020).

En la tabla 5, se presenta el análisis chi cuadrado entre la variable capacidad de agencia de autocuidado ASA y el tiempo de evolución de la enfermedad en pacientes con Diabetes mellitus 2, en la que se indica que el valor chi calculado con un valor de 0.000 es menor que el valor chi de la tabla con 0.05, implicando que existe una relación de dependencia entre las variables, lo que evidencia que el tiempo de evolución de la enfermedad afecta la capacidad de agencia del autocuidado.

\section{DISCUSIÓN}

El estudio de la relación entre los factores básicos condicionantes y evolución de la enfermedad en el autocuidado de los pacientes con diabetes mellitus tipo 2 de 90 pacientes con diabetes, estos indicaron tener capacidad de agencia de autocuidado suficiente, escenario aceptable frente a los resultados reportados por otros estudios como el presentado ${ }^{9}$ en el 2016, donde los pacientes diabéticos tipo 2 presentaron ausencia de autocuidado y bajo nivel de conocimientos sobre diabetes mellitus, por lo que se recomienda el diseño e implementación de programas educativos.

Es de señalar, que mantener una adecuada agencia de autocuidado aumenta las probabilidades de obtener y optimizar las conductas de salud, así como el desarrollo de una capacidad definida de autogestión para controlar la diabetes como enfermedad ${ }^{27}$ 
Revista Arbitrada Interdisciplinaria de Ciencias de la Salud. SALUD Y VIDA

Volumen 4. Número 8. Año 4. Julio - Diciembre 2020

Hecho el depósito de Ley: FA2016000010

ISSN: 2610-8038

FUNDACIÓN KOINONIA (F.K).

Santa Ana de Coro, Venezuela.

Franklin Antonio Vite-Solorzano; Leobaldo Barrera-Paz; Jefferson Alexander Sornoza-Párraga Angy Vanessa Salazar-Morrillo

28. Sin embargo, cabe señalar que aun cuando los pacientes manifiesten y/o se evalúen con un buen puntaje de capacidad de agencia de autocuidado, sus niveles de agencia no se reflejan en su quehacer.

La capacidad de agencia del autocuidado está siendo afectada por los factores básicos condicionantes de la edad, sexo, estado civil y el tiempo de evolución de la enfermedad 29. Mientras que los factores números de hijos, vivienda, religión y situación académica no presentaron relación de dependencia con el autocuidado, hecho que coincide parcialmente con los resultados presentados ${ }^{30}$ en el 2010, en el cual concluyo que la capacidad de autocuidado está vinculada a múltiples variables, que merecen atención de los profesionales de salud cuando se realiza la proposición de programas de educación. Se destaca el hecho, en la cual el cuidado de las personas con Diabetes Mellitus se ha convertido en un reto en los niveles de preparación del personal de salud para el cuidado de la misma, pero especialmente en los subcentros de zonas rurales de Manabí-Ecuador, pues su visión es fundamental para ayudar a los pacientes que sufren de esta enfermedad para lograr que alcancen una conducta de autocuidado mínima que les permita controlar la enfermedad y mantener una vida sana.

\section{CONCLUSIÓNES}

Existe una relación significativa de dependencia entre las variables autocuidado, factores condicionantes y evolución de la enfermedad, en la que se constató que el autocuidado puede variar o afectarse por factores condicionantes de la edad, sexo, estado civil y evolución de la enfermedad.

Asimismo, existe un total acuerdo entre los pacientes investigados, en considerar que poseen una buena capacidad de agencia de autocuidado, específicamente en lo que refieren a sus ajustes en los hábitos de vida para mantenerme saludable, en términos de limpieza e higiene personal, en dar prioridad a su enfermedad, en tratar de conocer acerca de su enfermedad, en mantener un constante monitoreo y solicitud de información 
Franklin Antonio Vite-Solorzano; Leobaldo Barrera-Paz; Jefferson Alexander Sornoza-Párraga Angy Vanessa Salazar-Morrillo

en caso de necesitarla y de mantener cuidado en caso de enfrentar problemas con la enfermedad. También, se determinó que los factores condicionantes del la edad, sexo, estado civil y evolución de la enfermedad afectan la capacidad de agencia de autocuidado ASA en pacientes con Diabetes mellitus 2.

\section{CONFLICTO DE INTERÉS}

Los autores declaran que no tienen conflicto de interés en la publicación de este artículo.

\section{FINANCIAMIENTO}

Autofinanciado.

\section{AGRADECIMIENTO}

A Universidad Técnica de Manabí, Portoviejo; por motivar el desarrollo la investigación.

\section{REFERENCIAS}

1. Rossaneis MA, Haddad Mdo C, Mathias TA, Marcon SS. Differences in foot selfcare and lifestyle between men and women with diabetes mellitus. Rev Lat Am Enfermagem. 2016 Aug 15;24:e2761. doi: 10.1590/1518-8345.1203.2761. PMID: 27533270; PMCID: PMC4996089.

2. Ferreira JSSP, Sacco ICN, Siqueira AA, Almeida MHM, Sartor CD. Rehabilitation technology for self-care: Customised foot and ankle exercise software for people with diabetes. PLoS One. 2019 Jun 20;14(6):e0218560. doi: 10.1371/journal.pone.0218560. PMID: 31220155; PMCID: PMC6586406.

3. Gil-Velázquez LE, Sil-Acosta MJ, Domínguez-Sánchez ER, Torres-Arreola Ldel P, Medina-Chávez JH. Guía de práctica clínica. Diagnóstico y tratamiento de la diabetes mellitus tipo 2. [Practice guideline. Diagnosis and treatment of type 2 diabetes mellitus]. Rev Med Inst Mex Seguro Soc. 2013 Jan-Feb;51(1):104-19. Spanish. PMID: 23550415. 
Franklin Antonio Vite-Solorzano; Leobaldo Barrera-Paz; Jefferson Alexander Sornoza-Párraga Angy Vanessa Salazar-Morrillo

4. Pérez Rodríguez Arnoldo, Barrios López Yusimí, Monier Tornés Aimeé, Berenguer Gouarnalusses Maritza, Martínez Fernández Irene. Repercusión social de la educación diabetológica en personas con diabetes mellitus. [Social repercussion of diabetes education in patients with diabetes mellitus]. MEDISAN [Internet]. $2009 ; 13(1)$.

5. Mendoza Irenia, Torres S José E, Herrera Ana, Colina Wilmer. Educación diabetológica y su proyección a la comunidad. [Diabetes education and its projection to the community]. Rev. Venez. Endocrinol. Metab. [Internet]. 2012; 10(Suppl 1): 138-142.

6. Péres DS, Santos MA, Zanetti ML, Ferronato AA. Dificuldades dos pacientes diabéticos para o controle da doença: sentimentos e comportamentos. [Difficulties two diabetic patients for or controle da doenca: sentiments and behaviors] Rev. Latino-Am. Enfermagem. 2007; 15(6): 1105-12.

7. Castillo-Guzmán S, Palacios-Ríos D, López-Cabrera NG, Hernández-Guedea MA, Rodríguez-Rodriguez NH, González-Santiago O. Seasonality variation of amputations in a university hospital from Monterrey, Mexico (2009-2012). [Variación estacional de las amputaciones en un hospital universitario de Monterrey, México (2009-2012)]. Cir Cir. 2018;86(5):399-403. Spanish. doi: 10.24875/CIRU.18000111. PMID: 30226498.

8. Hernández-Marín JD, Marrufo-Patrón CA, López-Rosales F. Metabolic changes in obese patients after laparoscopic gastric bypass: five-year experience in a tertiary referral hospital. [Efectos metabólicos en pacientes obesos posoperados de bypass gástrico laparoscópico: 5 años de experiencia en un hospital de tercer nivel]. Cir Cir. 2018;86(4):338-346. Spanish. doi: 10.24875/CIRU.M18000052. PMID: 30067711.

9. Organización Panamericana de Salud. Fortalecimiento del autocuidado como estrategia de la Atención Primaria en Salud: la contribución de las instituciones de salud en América Latina. [Strengthening self-care as a Primary Health Care Strategy: The Contribution of Health Institutions in Latin America]. Chile (Santiago): Organización Panamericana de la Salud; 2016. 
Franklin Antonio Vite-Solorzano; Leobaldo Barrera-Paz; Jefferson Alexander Sornoza-Párraga Angy Vanessa Salazar-Morrillo

10. Camarelles F, Tranche S, Muñoz E, Gómez JM. Semana del autocuidado semFYC [SemFYC Self-Care Week]. Aten Primaria. 2013 May;45(5):229-30. Spanish. doi: 10.1016/..aprim.2013.04.001. PMID: 23684376; PMCID: PMC6985513.

11. Martin-Morales A, Meijide Rico F, García González JI, Regadera Anechina L, Manero Font $M$. Repercusiones psicológicas de la disfunción eréctil sobre la autoestima y autoconfianza. [Psychological impact of erectile dysfunction on selfesteem and self-confidence]. Actas Urol Esp. 2005 May;29(5):493-8. Spanish. doi: 10.1016/s0210-4806(05)73280-0. PMID: 16013795.

12. Bernal BML, Ponce GG. Propuesta para la enseñanza del cuidado en Enfermería. [Proposal for the teaching of nursing care]. Enfermería Universitaria. 2009;6(1):3341.

13. Feo R, Kitson A, Conroy T. How fundamental aspects of nursing care are defined in the literature: A scoping review. J Clin Nurs. 2018 Jun;27(11-12):2189-2229. doi: 10.1111/jocn.14313. PMID: 29514402.

14. Dick TK, Patrician PA, Loan LA. The Value of Nursing Care: A Concept Analysis. Nurs Forum. 2017 Oct;52(4):357-365. doi: 10.1111/nuf.12204. Epub 2017 Apr 13. PMID: 28407260.

15. Wall ML. Contributions of nursing care to women. Rev Bras Enferm. 2018;71(suppl 3):1203-1204. English, Portuguese, Spanish. doi: 10.1590/0034-7167201871sup301. PMID: 29972515.

16. Velandia A, Rivera LN. Confiabilidad de la escala "Apreciación de la agencia de autocuidado" (ASA), segunda versión en español, adaptada para población colombiana. [Scale reliability "Self-care Agency Appreciation" (SAA), second version in Spanish, adapted for the Colombian population]. Av. enferm. [Internet]. 1 de enero de 2009 [citado 3 de diciembre de 2020];27(1):38-47.

17. Gao H, Söderhamn U, Cliffordson C, Guo L, Guo Q, Liu K. Reliability and validity of the Chinese version of the Self-care Ability Scale for the Elderly. J Clin Nurs. 2017 Dec;26(23-24):4489-4497. doi: 10.1111/jocn.13779. Epub 2017 Apr 20. PMID: 28231632. 
Franklin Antonio Vite-Solorzano; Leobaldo Barrera-Paz; Jefferson Alexander Sornoza-Párraga Angy Vanessa Salazar-Morrillo

18. Kav S, Yilmaz AA, Bulut Y, Dogan N. Self-efficacy, depression and self-care activities of people with type 2 diabetes in Turkey. Collegian. 2017;24(1):27-35. doi: 10.1016/j.colegn.2015.09.005. PMID: 29218959.

19. López Siguero JP. Riesgos metabólicos del consumo excesivo de bebidas con azúcares refinados. Nutr Hosp. 2016 Mar 25;33(2):89. Spanish. doi: 10.20960/nh.89. PMID: 27238772.

20. Leiva AM, Martínez MA, Cristi-Montero C, Salas C, Ramírez-Campillo R, Díaz Martínez X, Aguilar-Farías N, Celis-Morales C. El sedentarismo se asocia a un incremento de factores de riesgo cardiovascular y metabólicos independiente de los niveles de actividad física. [Sedentary lifestyle is associated with metabolic and cardiovascular risk factors independent of physical activity]. Rev Med Chil. 2017 Apr;145(4):458-467. Spanish. doi: 10.4067/S0034-98872017000400006. PMID: 28748993.

21. Wiedmer P, Nogueiras R, Broglio F, D'Alessio D, Tschöp MH. Ghrelin, obesity and diabetes. Nat Clin Pract Endocrinol Metab. 2007 Oct;3(10):705-12. doi: 10.1038/ncpendmet0625. PMID: 17893689.

22. Pajuelo Ramírez Jaime, Bernui Leo Ivonne, Sánchez González José, Arbañil Huamán Hugo, Miranda Cuadros Marianella, Cochachin Henostroza Omaira et al. Obesidad, resistencia a la insulina y diabetes mellitus tipo 2 en adolescentes. [Obesity, insulin resistance and type 2 diabetes mellitus in adolescents]. An. Fac. med. [Internet]. 2018; 79(3): 200-205.

23. Vigil-De Gracia P, Olmedo J. Diabetes gestacional: conceptos actuales. [Gestational diabetes: Current concepts]. Ginecol. obstet. Méx. [revista en la Internet]. 2017; 85(6): 380-390.

24. Soler Sánchez Y, Pérez R, López Sánchez M, Quezada Rodríguez D. Conocimientos y autocuidado en pacientes con diabetes mellitus tipo 2. [Knowledge and self-care in patients with type 2 diabetes]. AMC [Internet]. 2016; 20(3): 244-252.

25. Lázaro-Martínez JL, Tardáguila-García A, García-Klepzig JL. Diagnostic and therapeutic update on diabetic foot osteomyelitis. Endocrinol Diabetes Nutr. 2017 Feb;64(2):100-108. English, Spanish. doi: 10.1016/j.endinu.2016.10.008. Epub 2017 Feb 10. PMID: 28440774. 
Revista Arbitrada Interdisciplinaria de Ciencias de la Salud. SALUD Y VIDA

Volumen 4. Número 8. Año 4. Julio - Diciembre 2020

Hecho el depósito de Ley: FA2016000010

ISSN: 2610-8038

FUNDACIÓN KOINONIA (F.K).

Santa Ana de Coro, Venezuela.

Franklin Antonio Vite-Solorzano; Leobaldo Barrera-Paz; Jefferson Alexander Sornoza-Párraga Angy Vanessa Salazar-Morrillo

26. Winocour PH. Diabetes and chronic kidney disease: an increasingly common multimorbid disease in need of a paradigm shift in care. Diabet Med. 2018 Mar;35(3):300-305. doi: 10.1111/dme.13564. Epub 2018 Jan 8. PMID: 29247554.

27. Velandia-Arias A, Rivera-Alvarez LN. Agencia de Autocuidado y Adherencia al Tratamiento en Personas con Factores de Riesgo Cardiovascular. [Self-care agency and adherence to treatment in people having cardiovascular risk]. Rev Salud Publica (Bogota). 2009 Aug;11(4):538-48. Spanish. doi: 10.1590/s012400642009000400005. PMID: 20169210.

28. Rivera Álvarez LN. Capacidad de agencia de autocuidado en personas con Hipertensión arterial hospitalizadas en una clínica de Bogotá, Colombia. [Self-care agency ability in people suffering from high blood pressure hospitalised in Bogotá, Colombia]. Rev Salud Publica (Bogota). 2006 Sep-Dec;8(3):235-47. Spanish. doi: 10.1590/s0124-00642006000300009. PMID: 17269223.

29. Tarwirei T, Nkhoma G, Mukona D, Zvinavashe M. The relationship between perceived self-efficacy and adherence to self-care activities in type 2 diabetic clients. Cent Afr J Med. 2013 Jan-Apr;59(1-4):6-14. PMID: 29144115.

30. Baquedano Irasema Romero, Santos Manoel Antônio dos, Martins Tatiane Aparecida, Zanetti Maria Lúcia. Autocuidado de personas con Diabetes Mellitus atendidas en un servicio de urgencia en México. [Self-Care of Patients with Diabetes Mellitus Cared for at an Emergency Service in Mexico]. Rev. Latino-Am. Enfermagem [Internet]. 2010; 18(6): 1195-1202.

C2020 por los autores. Este artículo es de acceso abierto y distribuido según los términos y condiciones de la licencia Creative Commons Atribución-NoComercial-Compartirlgual 4.0 Internacional (CC BY-NC-SA 4.0)

(https://creativecommons.org/licenses/by-nc-sa/4.0/). 